\title{
Hepatitis E Virus Infection and Butchers: A Case-Control Seroprevalence Study
}

\author{
Cosme Alvarado-Esquivel ${ }^{\mathrm{a}, \mathrm{c}}$, Veronica Dayali Gutierrez-Martinez ${ }^{\mathrm{b}}$, \\ Eda Guadalupe Ramirez-Valles ${ }^{\mathrm{b}}$, Antonio Sifuentes-Alvarez ${ }^{\mathrm{a}}$
}

\begin{abstract}
Background: Very few case-control studies to assess the risk of hepatitis $\mathrm{E}$ virus (HEV) infection in meat workers have been published. Therefore, we sought to determine: 1) the association of HEV IgG seropositivity and the occupation of butcher; and 2) the sociodemographic, work, clinical and behavioral characteristics of butchers associated with HEV exposure.
\end{abstract}

Methods: We performed a case-control seroprevalence study of 101 butchers (mean age: $38.50 \pm 12.52$ years) and 101 age-, gender- and residence-matched control subjects of the general population. AntiHEV IgG antibodies were determined using a commercially available enzyme-linked immunoassay. Bivariate and regression analyses were used to assess the association between HEV seropositivity and characteristics of butchers.

Results: Anti-HEV IgG antibodies were found in 18 (17.8\%) of the 101 butchers and in $14(13.9 \%)$ of the 101 control subjects (odds ratio (OR): 1.34 ; 95\% confidence interval $(\mathrm{CI}): 0.63-2.88$; $\mathrm{P}=0.44$ ). Stratification by sex, age and area of residence (rural or urban) in cases and controls showed similar seroprevalences of HEV infection among groups. Bivariate analysis showed that HEV seroprevalence was associated with low education (up to 6 years), work place, seniority, eating while working, a history of raising farm animals and national trips. However, further analysis by logistic regression showed that only the variable of national trips was associated with HEV exposure (OR: 5.38; 95\% CI: 1.02 - 28.16; $\mathrm{P}=0.04$ ). Concerning clinical characteristics of butchers, no association between HEV exposure and health status, history of surgery or blood transfusion was found.

Conclusions: Results from this first age-, gender- and residence-

Manuscript submitted June 17, 2019, accepted July 11, 2019

Published online April 21, 2021

aBiomedical Research Laboratory, Faculty of Medicine and Nutrition, Juarez University of Durango State, Avenida Universidad S/N, 34000 Durango, Dgo, Mexico

bFaculty of Chemical Sciences, Juarez University of Durango State, Avenida Veterinarias S/N, 34120 Durango, Dgo, Mexico

${ }^{\mathrm{c} C}$ Corresponding Author: Cosme Alvarado-Esquivel, Biomedical Research Laboratory, Faculty of Medicine and Nutrition, Av. Universidad S/N, 34000 Durango, Dgo, Mexico. Email: alvaradocosme@yahoo.com

doi: https://doi.org/10.14740/gr1198 matched serosurvey of HEV infection in butchers in Mexico suggest that this population group does not have a higher risk for HEV infection than people from the general population. However, further studies to confirm the lack of association between HEV infection and the occupation of butcher are needed.

Keywords: Hepatitis E virus; Butchers; Epidemiology; Case-control study; Mexico

\section{Introduction}

Hepatitis $\mathrm{E}$ is one of the most frequent acute viral hepatitis worldwide [1]. This disease is caused by hepatitis $\mathrm{E}$ virus (HEV), an RNA virus of positive polarity [2]. About 20 million cases of acute hepatitis E occur yearly [3]. Infections with HEV may also lead to fulminant hepatitis and chronic hepatitis [4]. HEV infection has also been associated with maternal mortality [5]. Transmission routes of HEV vary depending of the development of the country. In developing countries, transmission of HEV occurs mainly by ingestion of contaminated drinking water with human feces [6], whereas in developed countries, infections with HEV occur by zoonotic transmission or transfusion of contaminated blood products [6]. Other routes of HEV transmission include solid organ transplantation [7] and hemodialysis [8]. Infections with HEV have been demonstrated in several animals used for human consumption including, for instance, pigs [9], wild boars [10], deer [11], goats [12, 13] and sheep [14]. Seroreactivity to an agent antigenically related to the HEV was found in dairy cows in the United States recently [15]. Exposure to meat from infected animals might be a risk for HEV infections in humans [16-18].

Very few studies on the seroepidemiology of HEV exposure in butchers have been reported. High seroprevalence of HEV exposure in butchers has been found, for instance, $57.7 \%$ in China [14] and 76\% in Burkina Faso [19]. However, there is a lack of age- and gender-matched case-control studies to assess the association between HEV exposure and a butcher occupation. Therefore, this study aimed to determine: 1) the association between anti-HEV IgG antibodies seropositivity and the occupation of butcher; and 2) the association between HEV seropositivity and the work characteristics of butchers. 


\section{Materials and Methods}

\section{Study design and populations studied}

We performed an age- and gender-matched case-control study of 101 butchers and 101 people without an occupation of butcher. Inclusion criteria for butchers (cases) were: 1) people with occupation of butchers; 2) working in the municipality of Durango, Mexico; and 3) aged 16 years and older. Of the 101 butchers, 17 were females and 84 males. Age in butchers varied from 16 to 71 (mean: $38.50 \pm 12.52$ ) years. Twentyfive butchers were enrolled in rural areas and 76 in an urban area (Durango City). Inclusion criteria for the control group were: 1) people without occupation of butcher; 2) residence in the municipality of Durango; and 3) aged 16 years and older. Control subjects were matched with cases by age, gender and area of residence (rural or urban). Thus, the control group included 17 females and 84 males with an age varying from 18 to 71 (mean: $38.43 \pm 14.13$ ) years. Twenty-five controls were enrolled in rural areas and 76 in an urban area (Durango City). Age, sex and residence area were similar in cases and controls $(\mathrm{P}=0.97, \mathrm{P}=1.00$ and $\mathrm{P}=1.00$, respectively $)$.

\section{Sociodemographic, work, behavioral and clinical charac- teristics of butchers}

We obtained the sociodemographic, work, behavioral and clinical characteristics of butchers with a standardized questionnaire. The sociodemographic characteristics included age, sex, birthplace, residence, education and socioeconomic status. Work characteristics included work place (butcher shop or abattoir), seniority (years working in the meat industry), frequency (number of days a week) of contact with raw meat, use of masks, gloves and goggles, history of splashes at face with raw meat or blood, eating when working and injuries with sharp material at work. Behavioral characteristics included consumption of untreated water, unpasteurized milk, unwashed raw fruits and vegetables, ham, salami or sausages, raw or undercooked meat and type of meat consumed (pork, boar, turkey, chicken, duck, beef, venison, lamb, goat, rabbit, horse, or other). In addition, a history of raising farm animals, frequency of eating away from home (in restaurants or fast food outlets), soil contact, type of flooring at home and national or international traveling was recorded. Clinical characteristics included health status, and history of surgery, organ transplant, or blood transfusion.

\section{Detection of anti-HEV IgG antibodies}

Serum was obtained from a 3-mL blood sample of each participant. Sera were kept frozen at $-20{ }^{\circ} \mathrm{C}$ until analyzed. Detection of anti-HEV IgG antibodies was performed by using the commercially available enzyme immunoassay kit "AccuDiagTM HEV IgG ELISA” (Diagnostic Automation Inc., Woodland Hills, CA, USA). All assays were performed following the manufacturer's instructions.

\section{Statistical analysis}

Data were analyzed with the software SPSS version 20 and Epi Info version 7 . The sample size was calculated using the following values: a power of $80 \%$, a $95 \%$ two-sided confidence level, a 1:1 ratio of cases and controls, a $36.6 \%$ outcome in unexposed group [20] and an odds ratio (OR) of 2.5. The result of this calculation was 86 cases and 86 controls. For age matching, we used the Student's $t$-test. The Pearson's Chi-squared test was used for matching of gender and area of residence and to compare the HEV IgG seropositivity rate in cases and controls. In addition, the Pearson's Chi-squared test or the two-tailed Fisher's exact test (for values $<5$ ) were used to assess the association between the characteristics of butchers and the presence of anti-HEV IgG antibodies. Logistic regression analysis with the Enter method was used to assess further the association between HEV seropositivity and sociodemographic, work and behavioral characteristics. Only characteristics that obtained a $\mathrm{P}$ value $<0.05$ in the bivariate analysis were analyzed by logistic regression. We calculated the OR and 95\% confidence interval (CI), and a $\mathrm{P}$ value less than 0.05 was considered as statistically significant.

This study was conducted in compliance with the ethical standards of the responsible institution on human subjects as well as with the Helsinki Declaration. The Ethical Committee of the Faculty of Medicine and Nutrition in Durango City, Mexico approved this study. Cases and controls were informed about the aims, purposes and procedures of the study before enrollment. All cases and controls voluntarily accepted to participate in the study.

\section{Results}

Anti-HEV IgG antibodies were found in 18 (17.8\%) of the 101 butchers and in $14(13.9 \%)$ of the 101 control subjects. Seroprevalence of HEV infection in butchers was similar to the one found in controls (OR: 1.34; 95\% CI: $0.63-2.88 ; \mathrm{P}=$ $0.44)$. Stratification by sex, age and area of residence in cases and controls showed similar seroprevalences of HEV infection among groups (Table 1). Of the sociodemographic characteristics of butchers, bivariate analysis showed that HEV seroprevalence was higher in butchers with low education (up to 6 years) than those with higher education (more than 6 years) $(\mathrm{P}=0.03)$ (Table 2). Other sociodemographic characteristics including age, sex, birthplace, residence and socioeconomic level did not show an association with HEV exposure. With respect to work characteristics, the variables of work place, seniority and eating while working showed $\mathrm{P}$ values $<0.05$ by bivariate analysis (Table 3 ). Whereas frequency of contact with raw meat, use of masks, gloves and goggles, history of splashes at face with raw meat or blood, and injuries with sharp material at work were not associated with HEV exposure ( $P$ value $>0.05)$. Concerning clinical characteristics of butchers, health status and history of surgery or blood transfusion were 
Table 1. Stratification by Sex, Age and Residence in Butchers and Controls and Seropositivity to HEV

\begin{tabular}{|c|c|c|c|c|c|c|c|c|c|}
\hline \multirow{3}{*}{ Characteristic } & \multicolumn{3}{|c|}{ Cases } & \multicolumn{3}{|c|}{ Controls } & \multirow{3}{*}{$\begin{array}{l}\text { Odds } \\
\text { ratio }\end{array}$} & \multirow{3}{*}{$\begin{array}{l}95 \% \text { confi- } \\
\text { dence interval }\end{array}$} & \multirow{3}{*}{ P value } \\
\hline & \multirow{2}{*}{ No. of tested } & \multicolumn{2}{|c|}{ Seropositivity to HEV } & \multirow{2}{*}{ No. of tested } & \multicolumn{2}{|c|}{ Seropositivity to HEV } & & & \\
\hline & & No. & $\%$ & & No. & $\%$ & & & \\
\hline Male & 84 & 17 & 20.2 & 84 & 13 & 15.5 & 1.38 & $0.62-3.07$ & 0.42 \\
\hline Female & 17 & 1 & 5.9 & 17 & 1 & 5.9 & 1.00 & $0.05-17.41$ & 1.00 \\
\hline $31-50$ & 52 & 10 & 19.2 & 45 & 6 & 13.3 & 1.54 & $0.51-4.65$ & 0.43 \\
\hline$>50$ & 20 & 6 & 30.0 & 24 & 6 & 25.0 & 1.28 & $0.34-4.86$ & 0.71 \\
\hline \multicolumn{10}{|l|}{ Residence } \\
\hline Urban & 76 & 12 & 15.8 & 76 & 4 & 5.3 & 3.37 & $1.03-10.99$ & 0.06 \\
\hline
\end{tabular}

HEV: hepatitis E virus.

not associated with HEV exposure. None of the butchers had had an organ transplantation. Of the behavioral characteristics, the variables of a history of raising farm animals and national trips were associated with HEV exposure by bivariate analysis. Whereas the rest of behavioral characteristics of butchers including consumption of untreated water, unpasteurized milk, unwashed raw fruits and vegetables, ham, salami or sausages, raw or undercooked meat, type of meat consumed, frequency of eating away from home, soil contact, and type of flooring at home did not show an association with HEV exposure. A correlation of a selection of behavioral variables and HEV seroprevalence is shown in Table 4 . Further analysis by using logistic regression of sociodemographic, work and behavioral characteristics of butchers with $\mathrm{P}$ values $<0.05$ obtained by bi-

Table 2. Sociodemographic Characteristics of Butchers and Seroprevalence of HEV Infection

\begin{tabular}{|c|c|c|c|c|}
\hline \multirow{2}{*}{ Characteristic } & \multirow{2}{*}{ No. of subjects tested } & \multicolumn{2}{|c|}{ Prevalence of $\mathrm{HEV}$ infection } & \multirow{2}{*}{ P value } \\
\hline & & No. & $\%$ & \\
\hline \multicolumn{5}{|l|}{ Age groups (years) } \\
\hline $31-50$ & 52 & 10 & 19.2 & \\
\hline$>50$ & 20 & 6 & 30.0 & \\
\hline Female & 17 & 1 & 5.9 & \\
\hline \multicolumn{5}{|l|}{ Birth place } \\
\hline Durango State & 92 & 15 & 16.3 & 0.15 \\
\hline Other Mexican State & 8 & 3 & 37.5 & \\
\hline \multicolumn{5}{|l|}{ Residence place } \\
\hline \multicolumn{5}{|l|}{ Educational level } \\
\hline Up to 6 years & 39 & 11 & 28.2 & 0.03 \\
\hline$>6$ years & 61 & 7 & 11.5 & \\
\hline \multicolumn{5}{|l|}{ Socio-economic level } \\
\hline Low & 35 & 9 & 25.7 & 0.08 \\
\hline Medium & 59 & 7 & 11.9 & \\
\hline
\end{tabular}

HEV: hepatitis E virus. 
Table 3. Bivariate Analysis of Work Factors and HEV Seropositivity in Butchers

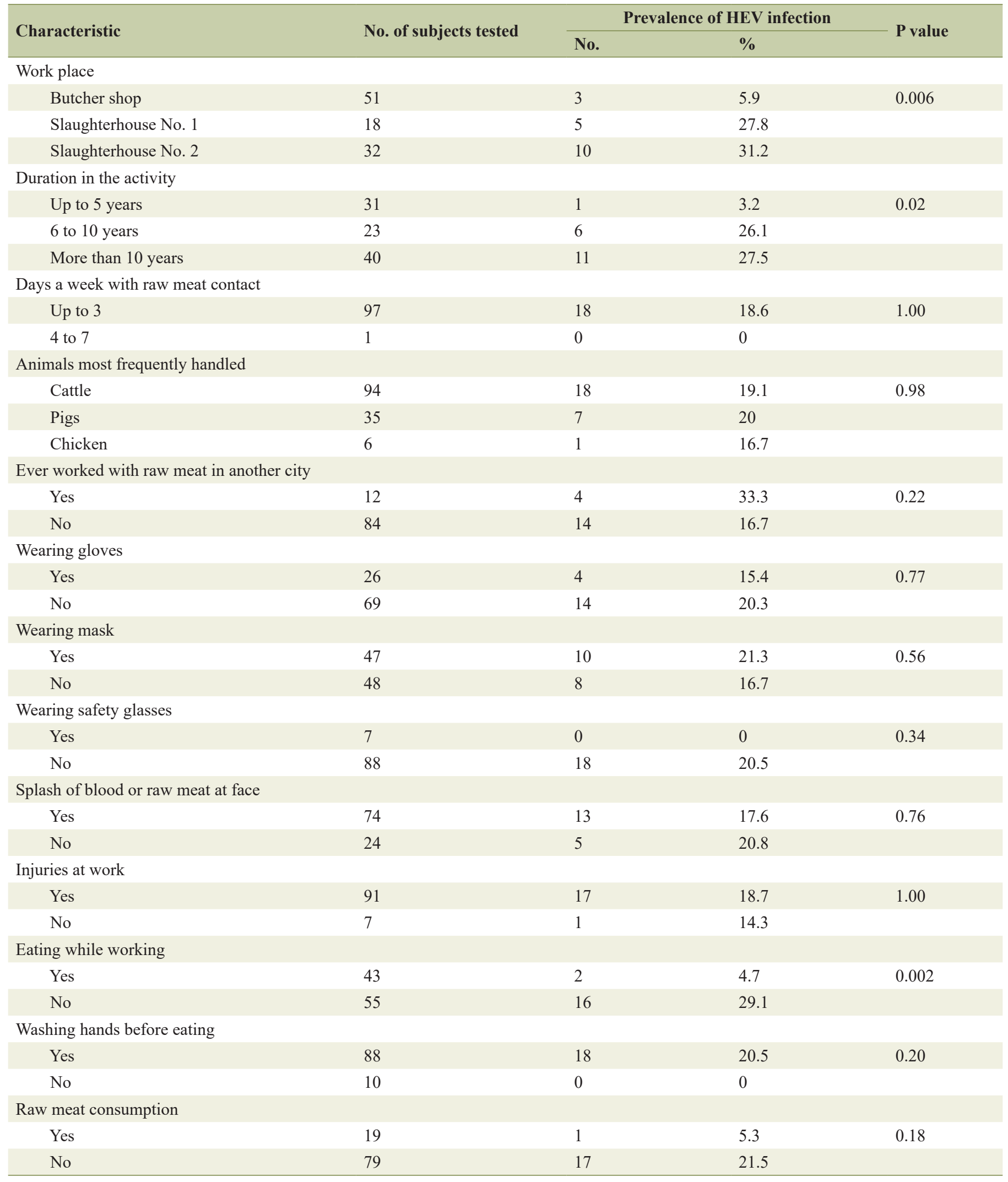

HEV: hepatitis E virus. 
Table 4. Bivariate Analysis of a Selection of Behavioral Factors and Seroprevalence of Infection With HEV in Butchers

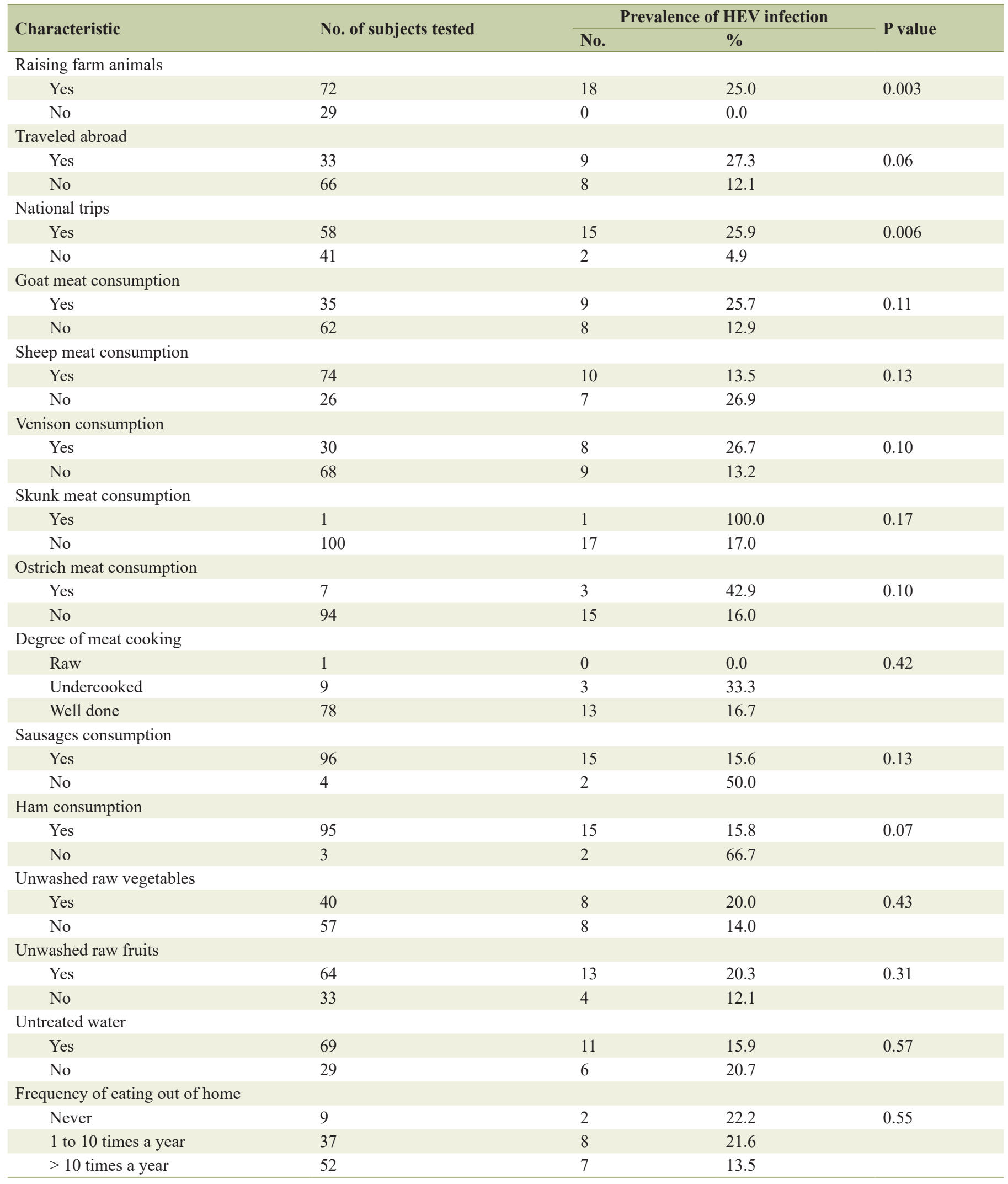

HEV: hepatitis E virus. 
Table 5. Multivariate Analysis of Selected Characteristics of Butchers and Their Association With HEV Infection

\begin{tabular}{llll}
\hline Characteristic & Odds ratio & 95\% confidence interval & P value \\
\hline Low education $(\leq 6$ years) & 1.00 & $0.27-3.68$ & 0.98 \\
Work place & 3.65 & $0.52-25.56$ & 0.19 \\
Seniority & 1.94 & $0.80-4.69$ & 0.13 \\
Eating while working & 2.07 & $0.27-15.51$ & 0.47 \\
Raising farm animals & 0.00 & 0.00 & 0.99 \\
National trips & 5.38 & $1.02-28.16$ & 0.04 \\
\hline
\end{tabular}

HEV: hepatitis E virus.

variate analysis showed that only the variable of national trips was associated with HEV exposure (OR: 5.38; 95\% CI: 1.02 - 28.16; $\mathrm{P}=0.04$ ) (Table 5).

\section{Discussion}

Hepatitis E is a zoonosis and contact with blood or flesh of animals with HEV infection may represent a risk for infection in humans. However, the magnitude of the association between occupational exposure to animal blood or flesh and HEV infection has been scantily studied. Therefore, in this study, we aimed to determine the association between HEV seropositivity and the occupation of butcher in a sample of people in Durango, Mexico. We found a similar seroprevalence of $\mathrm{HEV}$ infection in butchers than in controls. Even stratification by sex, age and area of residence in cases and controls showed similar seroprevalences of HEV infection among groups. Results thus indicate that the occupation of butcher does not represent a risk for HEV infection in our setting. The lack of association between HEV exposure and butcher occupation was unexpected. Butchers have more contact with blood and flesh of animals for human consumption than people without this occupation. And blood and flesh from many animals for human consumption might have HEV. In fact, HEV infection has been demonstrated in backyard and slaughtered pigs in our region [21]. Pork is commonly handled by butchers in our region. The seroprevalence (17.8\%) found in butchers is low as compared to that found in adults of the general population in rural Durango, Mexico (36.6\%) [20]. However, the seroprevalence found in butchers in this study is higher than those previously reported in other populations in rural Durango including pregnant women (5.7\%) [22] and Mennonites (6.7\%) [23]. In these studies $[20,22,23]$, the enzyme immunoassay for detection of $\mathrm{HEV}$ antibodies was the same as the one used in the present study. However, differences in age of individuals can explain the difference in seroprevalence found in butchers and pregnant women. Mean age in butchers was $38.50 \pm 12.52$ years, whereas mean age in pregnant women was $24.53 \pm 6.1$ years [22]. Seroprevalence of HEV exposure increases with age in our region [20, 22, 23] and in other countries [24, 25]. This was a reason we performed an age-matching in our study. The mean ages in the other population groups including Mennonites (38.40 \pm 15.53 years) [23], and general population in rural areas (39.85 \pm 17.15 years) [20] were similar to the one in butchers.
Therefore, factors other than age might have contributed for infection in those groups. We looked for risk factors associated with HEV exposure in butchers. Logistic regression of sociodemographic, work and behavioral characteristics of butchers showed that only the variable of national trips was associated with HEV exposure. This fact suggests that HEV exposure in butchers was acquired in Mexican States other than Durango State. This finding further supports that occupational exposure to meat does not represent a high risk for HEV infection in our setting. In a study in Cyprus, researchers also found a low seroprevalence of HEV in butchers and veterinarians (2\%) [26]. In contrast, our results conflict with those reported in a study in Portugal where researchers found a higher seroprevalence of HEV infection in workers occupationally exposed to swine (slaughterhouse workers, veterinarians, butchers and pig farmers) than control individuals from the general population [27]. In another study, researchers found a higher seroprevalence of $\mathrm{HEV}$ infection in pork butchers than in controls of the general population in Burkina Faso [19]. However, in those studies, no age-, gender- and residence-matching was performed or reported. Age-matching is important because HEV exposure increases with age [20, 22-25]. In the present study, we also matched cases with controls for gender and residence. Significantly higher seroprevalence rates of HEV infection were found in residents of suburban and rural areas compared with residents of urban areas in a study in Croatia [28].

The limitations of the present study include: 1) studied butchers were enrolled in only one Mexican state; and 2) no further tests as anti-HEV IgM antibody reactivity or detection of RNA by molecular assays were performed.

\section{Conclusions}

Results from this first age-, gender- and residence-matched serosurvey of HEV infection in butchers in Mexico suggest that this population group does not have a higher risk for HEV infection than people from the general population. However, further studies to confirm the lack of association between HEV infection and the occupation of butcher are needed.

\section{Acknowledgments}

None to declare. 


\section{Financial Disclosure}

This study was financially supported by Juarez University of Durango State, Mexico.

\section{Conflict of Interest}

None to declare.

\section{Informed Consent}

A written informed consent was obtained from each participant.

\section{Author Contributions}

CAE designed the protocol, obtained the blood samples, performed the data analysis and wrote the manuscript. VDGM and EGRV performed the laboratory tests and data analysis. ASA performed the data analysis and reviewed the manuscript.

\section{Data Availability}

The authors declare that data supporting the findings of this study are available within the article.

\section{References}

1. Pisano MB, Martinez-Wassaf MG, Mirazo S, Fantilli A, Arbiza J, Debes JD, Re VE. Hepatitis E virus in South America: The current scenario. Liver Int. 2018;38(9):1536-1546.

2. Aggarwal R, Goel A. Advances in hepatitis E - I: virology, pathogenesis and diagnosis. Expert Rev Gastroenterol Hepatol. 2016;10(9):1053-1063.

3. Blasco-Perrin H, Abravanel F, Blasco-Baque V, Peron JM. Hepatitis E, the neglected one. Liver Int. 2016;36(Suppl 1):130-134.

4. Okamoto H. [Hepatitis $\mathrm{E}$ in patients with hematological disorders]. Rinsho Ketsueki. 2018;59(10):1914-1923.

5. Gurley ES, Hossain MJ, Paul RC, Sazzad HM, Islam MS, Parveen S, Faruque LI, et al. Outbreak of hepatitis $\mathrm{E}$ in urban Bangladesh resulting in maternal and perinatal mortality. Clin Infect Dis. 2014;59(5):658-665.

6. Kamar N, Izopet J, Pavio N, Aggarwal R, Labrique A, Wedemeyer H, Dalton HR. Hepatitis E virus infection. Nat Rev Dis Primers. 2017;3:17086.

7. Fang SY, Han H. Hepatitis E viral infection in solid organ transplant patients. Curr Opin Organ Transplant. 2017;22(4):351-355.

8. Haffar S, Bazerbachi F, Leise MD, Dillon JJ, Albright RC, Murad MH, Kamath PS, et al. Systematic review with meta-analysis: the association between hepatitis $\mathrm{E}$ seroprevalence and haemodialysis. Aliment Pharmacol Ther. 2017;46(9):790-799.

9. Khounvisith V, Tritz S, Khenkha L, Phoutana V, Keosengthong A, Pommasichan S, Nouanthong P, et al. High circulation of Hepatitis $\mathrm{E}$ virus in pigs and professionals exposed to pigs in Laos. Zoonoses Public Health. 2018;65(8):1020-1026.

10. De Sabato L, Ostanello F, De Grossi L, Marcario A, Franzetti B, Monini M, Di Bartolo I. Molecular survey of HEV infection in wild boar population in Italy. Transbound Emerg Dis. 2018;65(6):1749-1756.

11. Spancerniene U, Grigas J, Buitkuviene J, Zymantiene J, Juozaitiene V, Stankeviciute M, Razukevicius D, et al. Prevalence and phylogenetic analysis of hepatitis E virus in pigs, wild boars, roe deer, red deer and moose in Lithuania. Acta Vet Scand. 2018;60(1):13.

12. Di Martino B, Di Profio F, Melegari I, Sarchese V, Robetto S, Marsilio F, Martella V. Detection of hepatitis E virus (HEV) in goats. Virus Res. 2016;225:69-72.

13. Sanford BJ, Emerson SU, Purcell RH, Engle RE, Dryman BA, Cecere TE, Buechner-Maxwell V, et al. Serological evidence for a hepatitis e virus-related agent in goats in the United States. Transbound Emerg Dis. 2013;60(6):538545.

14. Wu J, Si F, Jiang C, Li T, Jin M. Molecular detection of hepatitis E virus in sheep from southern Xinjiang, China. Virus Genes. 2015;50(3):410-417.

15. Yugo DM, Cossaboom CM, Heffron CL, Huang YW, Kenney SP, Woolums AR, Hurley DJ, et al. Evidence for an unknown agent antigenically related to the hepatitis E virus in dairy cows in the United States. J Med Virol. 2019;91(4):677-686.

16. Serracca L, Battistini R, Rossini I, Mignone W, Peletto $\mathrm{S}$, Boin $\mathrm{C}$, Pistone $\mathrm{G}$, et al. Molecular Investigation on the Presence of Hepatitis E Virus (HEV) in Wild Game in North-Western Italy. Food Environ Virol. 2015;7(3):206212.

17. Aprea G, Amoroso MG, Di Bartolo I, D'Alessio N, Di Sabatino D, Boni A, Cioffi B, et al. Molecular detection and phylogenetic analysis of hepatitis E virus strains circulating in wild boars in south-central Italy. Transbound Emerg Dis. 2018;65(1):e25-e31.

18. Dremsek P, Wenzel JJ, Johne R, Ziller M, Hofmann J, Groschup $\mathrm{MH}$, Werdermann S, et al. Seroprevalence study in forestry workers from eastern Germany using novel genotype 3- and rat hepatitis E virus-specific immunoglobulin G ELISAs. Med Microbiol Immunol. 2012;201(2):189-200.

19. Traore KA, Ouoba JB, Huot N, Rogee S, Dumarest M, Traore AS, Pavio N, et al. Hepatitis E Virus Exposure is Increased in Pork Butchers from Burkina Faso. Am J Trop Med Hyg. 2015;93(6):1356-1359.

20. Alvarado-Esquivel C, Sanchez-Anguiano LF, HernandezTinoco J. Seroepidemiology of hepatitis e virus infection in general population in rural durango, Mexico. Hepat Mon. 2014;14(6):e16876.

21. Alvarado-Esquivel C, Sanchez-Anguiano LF, HernandezTinoco J, Alvarado-Felix AO. Seroepidemiology of hepatitis E virus infection in pigs in Durango State, Mexico. 
J Med Virol. 2018;90(2):328-333.

22. Alvarado-Esquivel C, Sanchez-Anguiano LF, HernandezTinoco J. Hepatitis E virus exposure in pregnant women in rural Durango, Mexico. Ann Hepatol. 2014;13(5):510517.

23. Alvarado-Esquivel C, Sanchez-Anguiano LF, HernandezTinoco J. Seroepidemiology of hepatitis e virus infection in mennonites in Mexico. J Clin Med Res. 2015;7(2):103108.

24. Olsoy IB, Henriksen S, Weissbach FH, Larsen M, Borgen K, Abravanel F, Kamar N, et al. Seroprevalence of hepatitis $\mathrm{E}$ virus (HEV) in a general adult population in Northern Norway: the Tromso study. Med Microbiol Immunol. 2019;208(6):715-725.

25. Dimeglio C, Beau F, Broult J, Gouy P, Izopet J, Lastere S,
Abravanel F. Hepatitis E prevalence in French Polynesian blood donors. PLoS One. 2018;13(12):e0208934.

26. Suer K, Guvenir M, Aykac A. A Special Risk Group for Hepatitis E Infection: The First Record of North Cyprus. Pol J Microbiol. 2018;67(4):525-528.

27. Teixeira J, Mesquita JR, Pereira SS, Oliveira RM, AbreuSilva J, Rodrigues A, Myrmel M, et al. Prevalence of hepatitis E virus antibodies in workers occupationally exposed to swine in Portugal. Med Microbiol Immunol. 2017;206(1):77-81.

28. Vilibic-Cavlek T, Vilibic M, Kolaric B, Jemersic L, Kucinar J, Barbic L, Bagaric A, et al. Seroepidemiology of hepatitis E in Selected population groups in Croatia: a prospective pilot study. Zoonoses Public Health. 2016;63(6):494-502. 